Ingo Gottschalk ${ }^{\mathrm{a}, \star}$, Judith S. Abel ${ }^{\mathrm{a}}$, Tina Menzel, Ulrike Herberg, Johannes Breuer, Ulrich Gembruch, Annegret Geipel, Konrad Brockmeier, Christoph Berg and Brigitte Strizek

\title{
Prenatal diagnosis, associated findings and postnatal outcome of fetuses with double outlet right ventricle (DORV) in a single center
}

https://doi.org/10.1515/jpm-2018-0316

Received September 18, 2018; accepted November 26, 2018; previously published online December 20, 2018

\section{Abstract}

Objective: To assess the spectrum of associated anomalies, the intrauterine course, postnatal outcome and management of fetuses with double outlet right ventricle (DORV).

Methods: All cases of DORV diagnosed prenatally over a period of 8 years were retrospectively collected in a single tertiary referral center. All additional prenatal findings were assessed and correlated with the outcome. The accuracy of prenatal diagnosis was assessed.

Results: Forty-six cases of DORV were diagnosed prenatally. The mean gestational age at first diagnosis was $21+4$ weeks (range, 13-37). A correct prenatal diagnosis of DORV was made in $96.3 \%$ of the cases. If the relation of the great arteries, the position of the ventricular septal defect (VSD) and additional cardiac anomalies are taken into account, the prenatal diagnosis was correct in $92.6 \%$ of the cases. One case was postnatally classified as transposition of the great arteries with subpulmonary VSD and was excluded from further analysis. A total of 41 (91.1\%) fetuses with DORV had major additional cardiac

\footnotetext{
ango Gottschalk and Judith S. Abel contributed equally to this work. *Corresponding author: Dr. med. Ingo Gottschalk, Bereich für Pränatale Medizin und Gynäkologische Sonographie, Universitätsfrauenklinik Köln, Kerpenerstr. 34, 50931 Cologne, Germany; and Division of Prenatal Medicine, University of Cologne, Cologne, Germany, E-mail: ingo.gottschalk@uk-koeln.de Judith S. Abel and Tina Menzel: Division of Prenatal Medicine, University of Cologne, Cologne, Germany Ulrike Herberg and Johannes Breuer: Department of Pediatric Cardiology, University of Bonn, Bonn, Germany Ulrich Gembruch, Annegret Geipel and Brigitte Strizek: Department of Obstetrics and Prenatal Medicine, University of Bonn, Bonn, Germany

Konrad Brockmeier: Department of Pediatric Cardiology, University of Cologne, Cologne, Germany Christoph Berg: Division of Prenatal Medicine, University of Cologne, Cologne, Germany; and Department of Obstetrics and Prenatal Medicine, University of Bonn, Bonn, Germany
}

anomalies, 30 (66.7\%) had extracardiac anomalies and 13 (28.9\%) had chromosomal or syndromal anomalies. Due to their complex additional anomalies, five (11.1\%) of our 45 fetuses had multiple malformations and were highly suspicious for non-chromosomal genetic syndromes, although molecular diagnosis could not be provided. Disorders of laterality occurred in $10(22.2 \%)$ fetuses. There were 17 terminations of pregnancy $(37.8 \%)$, two $(4.4 \%)$ intrauterine and seven (15.6\%) postnatal deaths. Nineteen of $22(86.4 \%)$ live-born children with an intention to treat were alive at last follow-up. The mean follow-up among survivors was 32 months (range, 2-72). Of 21 children who had already undergone postnatal surgery, eight (38.1\%) achieved biventricular repair and 13 (61.9\%) received univentricular palliation. One recently born child is still waiting for surgery. All children predicted prenatally to need a single ventricle palliation, and all children predicted to achieve biventricular repair, ultimately received the predicted type of surgery. After surgery, 14 of 18 (77.8\%) children were healthy without any impairment.

Conclusion: DORV is a rare and often complex cardiac anomaly that can be diagnosed prenatally with high precision. DORV is frequently associated with major additional anomalies, leading to a high intrauterine and postnatal loss rate due to terminations or declined postnatal therapy. Without additional anomalies, the prognosis is good, although approximately $60 \%$ of children will have single ventricle palliation.

Keywords: congenital heart defect; double outlet right ventricle; fetus; prenatal diagnosis.

\section{Introduction}

Double outlet right ventricle (DORV) is a rare and complex conotruncal malformation representing less than $1 \%$ of all congenital heart defects $[1,2]$. The first description by Witham defined DORV as both pulmonary artery and aorta originating from a morphological right ventricle [3]. Drainage of the left ventricle is commonly achieved 
through a ventricular septal defect (VSD) at different locations and with varying relation to the pulmonary or aortal outflow tract [4]. Other authors defined DORV only by the type of ventriculo-arterial connection as one of the great arteries overriding the VSD by more than 50\% [5-7].

To distinguish DORV from other conotruncal malformations, description of the relationship of the great arteries and the position of the VSD is crucial [8]. While DORV describes the type of ventriculo-arterial connection, tetralogy of Fallot (TOF) describes the infundibular morphology. Independent of any additional infundibular stenosis, the ventriculo-arterial connection of a case of TOF with more than $50 \%$ aortic overriding is unequivocally a DORV.

The postnatal physiology is mainly dependent on the location of the VSD in relation to the great arteries and the presence or absence of outflow tract obstructions. Severe pulmonary stenosis may lead to neonatal cyanosis whereas severe aortic stenosis or coarctation may necessitate prostaglandin $\mathrm{E}_{1}$ treatment in order to maintain adequate systemic perfusion. Therefore, accurate prenatal echocardiographic diagnosis is mandatory in counseling parents with regard to prognosis and treatment options as well as planning for delivery and postnatal management $[2,9]$.

DORV is frequently associated with additional major cardiac and extracardiac anomalies with an incidence of up to $90 \%$ in pre- and postnatal series [1, 2, 4, 7, 9-14]. In addition to the complex abnormal cardiac morphology itself in some types of DORV, associated cardiac anomalies are left and right ventricular outflow tract obstructions (LVOTO and RVOTO), atrioventricular (AV)-valve stenosis and atresia, hypoplastic left ventricle (HLV), straddling of aortic valve and coronary artery anomalies. Associated chromosomal anomalies including trisomy 13, trisomy 18 and 22q11 microdeletion occur in $25.0 \%$ to $45.2 \%$ of fetuses; all fetuses had additional cardiac or extracardiac anomalies [2, 9, 15-18]. Those additional anomalies significantly contribute to the postnatal mortality and morbidity and may complicate the surgical course.

Without additional coexisting anomalies, the outcome is usually good. Whether the subtype of DORV significantly influences the outcome is yet unclear. However, DORV may require more extensive surgery due to the great variations in abnormal cardiac morphology in comparison with TOF $[1,19]$.

The aim of this study was to assess the spectrum of associated cardiac, extracardiac and chromosomal anomalies, the intrauterine course and postnatal outcome of fetuses with DORV. The morphological variants and the accuracy of prenatal echocardiographic diagnosis of
DORV including VSD position, relationship of the great arteries and outflow tract obstructions were assessed.

\section{Materials and methods}

All prenatally diagnosed DORV were retrospectively reviewed for intrauterine course and outcome in the perinatal database of one tertiary referral center for prenatal medicine and fetal echocardiography (University of Cologne, Germany). All fetuses with DORV were diagnosed between January 2010 and December 2017.

The anatomic survey and fetal echocardiography were performed in a standardized fashion. Fetal echocardiography was carried out by a segmental approach using standardized anatomical planes incorporating pulsed-wave and color Doppler imaging $[20,21]$. Sector or curved array-probes of $5 \mathrm{MHz}, 7.5 \mathrm{MHz}$ or $9 \mathrm{MHz}$ were used for all ultrasound examinations (ATL HDI 5000 and IU22 Philips, Hamburg, Germany; Voluson 730 Pro and Expert, E8 and E10, respectively, GE Healthcare, Solingen, Germany). A pediatric cardiologist attended at least one of the prenatal ultrasound examinations and subsequently counseled the patients. Following delivery and initial care by the attending neonatologist, all newborns were examined by a pediatric cardiologist within $12 \mathrm{~h}$ after birth. Conventional karyotyping was performed in all cases, predominantly prenatally, otherwise postnatally. In two cases with normal karyotype, clinical anomalies prompted a targeted mutation analysis postnatally (Cornelia-de-Lange syndrome and Johanson-Blizzard syndrome).

In this study, DORV was primarily defined by the type of ventriculo-arterial connection: the origin of $50 \%$ or more of the circumference of each great artery was above the morphological right ventricle. Cases with an aortic overriding of 50\% and more and additional infundibular pulmonary stenosis were defined as the DORV Fallot type, whereas cases with infundibular stenosis and an aortic overriding of less than 50\% were classified as TOF. All cases with a postnatal diagnosis of TOF or TGA instead of DORV were excluded from the study. Only for the assessment of diagnostic accuracy, all newborns were included in the cohort as examples of incorrect diagnoses.

We classified different types of DORV in accordance with the current classification of the Society of Thoracic Surgeons-European Association for Cardiothoracic Surgery (STS-EACTS) International Nomenclature and the Association for European Pediatric Cardiology (AEPC) which defined four types of DORV $[7,14]$. Due to the high incidence of DORV in heterotaxy, we added a fifth type of DORV, the AVSD type in heterotaxy, as proposed by Lacour-Gayet [10].

(1) DORV, VSD type: normally related great arteries and aorta overriding the subaortic or doubly committed VSD by more than $50 \%$. (2) DORV, Fallot type: VSD type with additional RVOTO. (3) DORV, TGA type (Taussig-Bing): side-by-side related great arteries with subpulmonary VSD and overriding pulmonary trunk. (4) DORV non-committed VSD type: any relationship of great arteries and noncommitted (remote) VSD, mostly in hearts with AVSD. The VSD is far removed from both the aortic and pulmonary valves and is located within the inlet septum without perimembranous extension. (5) DORV, AVSD/heterotaxy type: DORV with AVSD and heterotaxy syndrome (either left or right isomerism).

The following variables were assessed, as far as retrospectively achievable: maternal age, gestational age at diagnosis, associated 
cardiac, extracardiac and chromosomal or genetic abnormalities, DORV classification according to the relationship of the great arteries, position of the VSD and presence or absence of outflow tract obstructions. Minor anomalies like right aortic arch, persistent left superior vena cava and singular umbilical artery were documented, but not classified as associated cardiac and extracardiac anomalies, respectively.

All data were retrieved from medical files, stored ultrasound images and, if available, from ultrasound video recordings, and preand postnatal diagnoses of all live-born children were compared to assess the accuracy of prenatal diagnosis.

All cases were classified into five groups according to the pregnancy outcome: termination of pregnancy (TOP), intrauterine fetal (IUFD) or neonatal death (NND), death in infancy or childhood (CHD) and survivors. Neonatal death was defined as death within the first 28 days of life, and CHD as any death after 28 days of life. Postnatal medical files of echocardiography, cardiac catheterization, surgery or autopsy were available for confirmation of the prenatal diagnosis in all live-born children.

Intergroup comparisons were made using the $\chi^{2}$ test, Student's $t$-test or Fisher's exact test when appropriate. Values are given as mean \pm standard deviation (SD) unless otherwise indicated. $\mathrm{P}<0.05$ was considered statistically significant. The Institutional Review Board of our center does not require formal approval for retrospective archive studies; therefore, ethical approval was not sought.

\section{Results}

During the study period, 46 cases of fetal DORV were diagnosed prenatally. The mean gestational age at first diagnosis was $21+4$ weeks (range, 13-37).

One case was postnatally classified as TGA with VSD. This case was excluded from the outcome analysis. Only for the assessment of diagnostic accuracy, all 46 cases were included.

Two (4.4\%) of the 45 fetuses had a prenatal diagnosis of VSD-type DORV, five (11.1\%) had the Fallot type, 27 (60.0\%) had the TGA type, three (6.7\%) had the non-committed VSD type and eight (17.8\%) had the AVSD type with heterotaxy.

\section{Additional cardiac, extracardiac and genetic anomalies}

Forty-one (91.9\%) fetuses with DORV had major additional cardiac anomalies. The most common cardiac anomalies were RVOTO in 15 (33.3\%) and LVOTO in 11 (24.4\%) cases. The remaining fetuses had HLV with or without mitral valve atresia (MA) in $13(28.9 \%)$ cases. Major extracardiac anomalies occurred in 30 (66.7\%) fetuses, including skeletal or facial dysmorphias, facial clefts, cerebral anomalies and bilateral renal agenesis. Disorders of laterality occurred in 10 (22.2\%) cases, including five cases with left isomerism, four cases with right isomerism and one case with situs inversus totalis. Chromosomal or nonchromosomal syndromes occurred in 13 (28.9\%) fetuses (trisomy 18 in six fetuses, trisomy 13, trisomy 17-mosaicism, triploidy, microdeletion 22q11, Kleefstra syndrome, Cornelia de Lange syndrome and Johanson-Blizzard syndrome in one fetus each). Due to their complex additional anomalies, five (11.1\%) other fetuses were highly suspicious for non-chromosomal genetic syndromes, but molecular diagnosis could not be provided.

\section{Outcome}

TOP was performed in 17 of the 45 (37.8\%) cases (Table 1 and Figure 1). All of these fetuses had additional major anomalies including eight fetuses with chromosomal anomalies and three fetuses which were highly suspicious for genetic syndromes due to additional facial, cerebral and skeletal anomalies.

IUFD occurred in two (4.4\%) fetuses with the AVSDtype DORV, left isomerism and complete heart block.

Twenty-six neonates $(57.8 \%)$ were born alive. NND occurred in three $(6.7 \%)$ cases, including one neonate with trisomy 18 and another one with a suspected genetic syndrome. Both neonates died after compassionate care. The third neonate died due to low cardiac output failure after cardiac surgery at the age of 15 days.

CHD occurred in four (8.9\%) cases, including two cases with trisomy 17 mosaicism and Cornelia de Lange syndrome, respectively. Both received compassionate

Table 1: Outcome of 45 cases with DORV according to the associated condition.

\begin{tabular}{lrrrrrr}
\hline & $\mathbf{n}$ & TOP & IUFD & NND & CHD & Alive \\
\hline Trisomy 18 & 6 & 5 & 1 & & \\
Trisomy 13 & 1 & 1 & & & & \\
Trisomy 17 mosaicism & 1 & & & & 1 & \\
Triploidy 69XXX & 1 & 1 & & & & \\
22q11 microdeletion & 1 & 1 & & & & \\
Cornelia de Lange syndrome & 1 & & & & 1 & \\
Kleefstra syndrome & 1 & & & & & 1 \\
Johanson-Blizzard syndrome & 1 & & & & & 1 \\
Multiple malformation syndrome & 7 & 5 & & 1 & & 1 \\
Heterotaxy & 10 & 2 & 2 & & & 6 \\
Isolated & 15 & 2 & & 1 & 2 & 10 \\
Total & 45 & 17 & 2 & 3 & 4 & 19 \\
\hline
\end{tabular}

TOP, termination of pregnancy; IUFD, intrauterine fetal death; NND, neonatal death; CHD, death in infancy or childhood. 


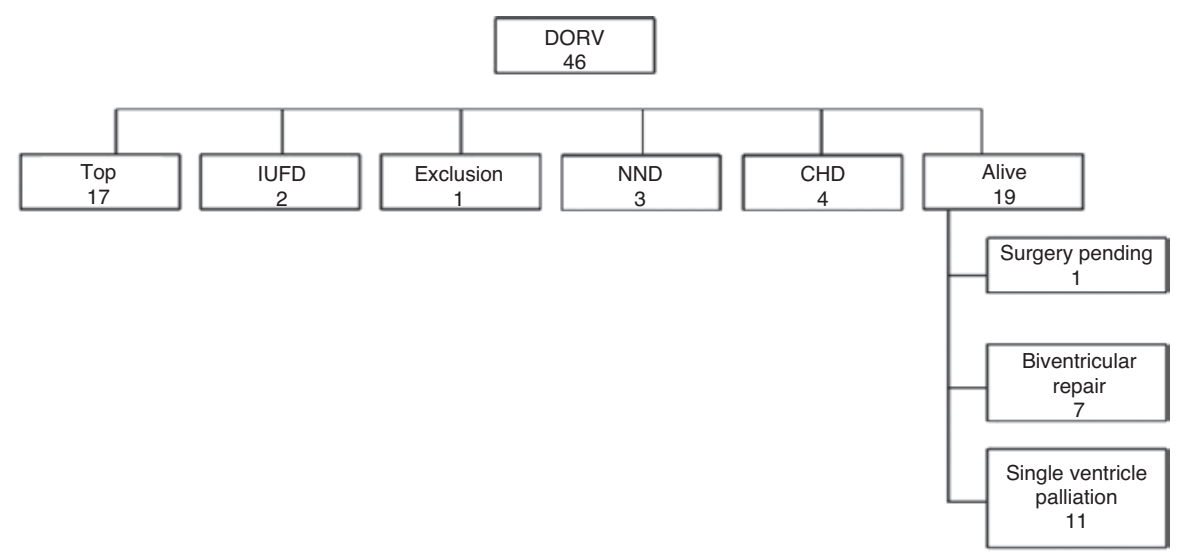

Figure 1: Outcome of 46 cases with prenatally diagnosed double outlet right ventricle (DORV).

TOP, termination of pregnancy; IUFD, intrauterine fetal death; NND, neonatal death; CHD, death in infancy or childhood.

care. The third infant died because of global respiratory insufficiency after cardiac surgery at the age of 3 months and the fourth infant died because of renal and cardiac failure after the second step of Norwood palliation at the age of 11 months (Table 1).

Nineteen of the 45 children with a confirmed diagnosis of DORV were alive at the time of writing of the manuscript, resulting in an overall survival rate of $42.2 \%$. After exclusion of TOP, IUFD and cases in which parents opted for compassionate care, the "intention-to-treat group" consisted of 22 fetuses, all were born alive (Table 2). Twenty-one of those infants already underwent surgery, three died and 18 survived. One infant is still awaiting surgery and facing univentricular palliation due to HLV and MA. Due to inadequate postnatal growth (5 kg body weight at the age of 7 months), repair of anomalous pulmonary venous connection and the first step of palliation were postponed. The mean follow-up of survivors was 32 months (range, 2-72).

The intention-to-treat survival rate was $86.4 \%$ (19/22). After surgery, $77.8 \%(14 / 18)$ were healthy without any impairment at the latest follow-up, 22.2\% (4/18) were mildly impaired (Figure 2).

\section{Postnatal cardiac surgery}

The type of postnatal surgery was dependent on the complexity of the cardiac defect itself and the postnatal hemodynamic situation. If surgical risk appeared to be too high or if biventricular complete repair did not seem achievable, univentricular palliation was performed. Eight of 21 (38.1\%) infants underwent biventricular repair and 13 of $21(61.9 \%)$ received univentricular palliation (Table 2). All children predicted prenatally to need a single ventricle palliation, and all children predicted to achieve biventricular repair, ultimately received the predicted type of surgery.

Within the biventricular repair group, infants received either one-stage complete repair or aorto-pulmonary (AP) shunting, right-ventricle-to-pulmonaryartery (RV-PA) conduit, VSD patch or VSD tunneling, aortic arch reconstruction, banding of pulmonary arteries or arterial switch operation, depending on their type of DORV and their postnatal hemodynamic situation. One of those eight infants had an additional coronary artery anomaly and died at the age of 15 days because of low cardiac output failure after aortic arch reconstruction and banding of pulmonary arteries. The other seven infants survived surgery, six were healthy without impairment and one infant was mildly impaired due to mild cardiac insufficiency (Table 2).

Univentricular palliation was performed in the presence of major additional cardiac anomalies, including MA, HLV, L-transposition of great arteries or severe obstruction of right and left ventricular outflow tract, respectively. Two of those 13 children died in childhood. The first child had complex DORV with MA, single right ventricle and interrupted aortic arch and died after the first step of Norwood palliation and aortic arch reconstruction because of global respiratory insufficiency at the age of 3 months. The second severely growth restricted child had TGA (Taussig-Bing)-type DORV with additional hypoplastic aortic arch and died at the age of 11 months after the second step of Norwood palliation because of pulmonary hypertension and renal and cardiac failure. The other 11 children survived, and eight were healthy without impairment. One child developed moderate pulmonary valve insufficiency and mild dyspnea, one child had autism-spectrum disorder and one child had concomitant Johanson-Blizzard syndrome (Table 2). 


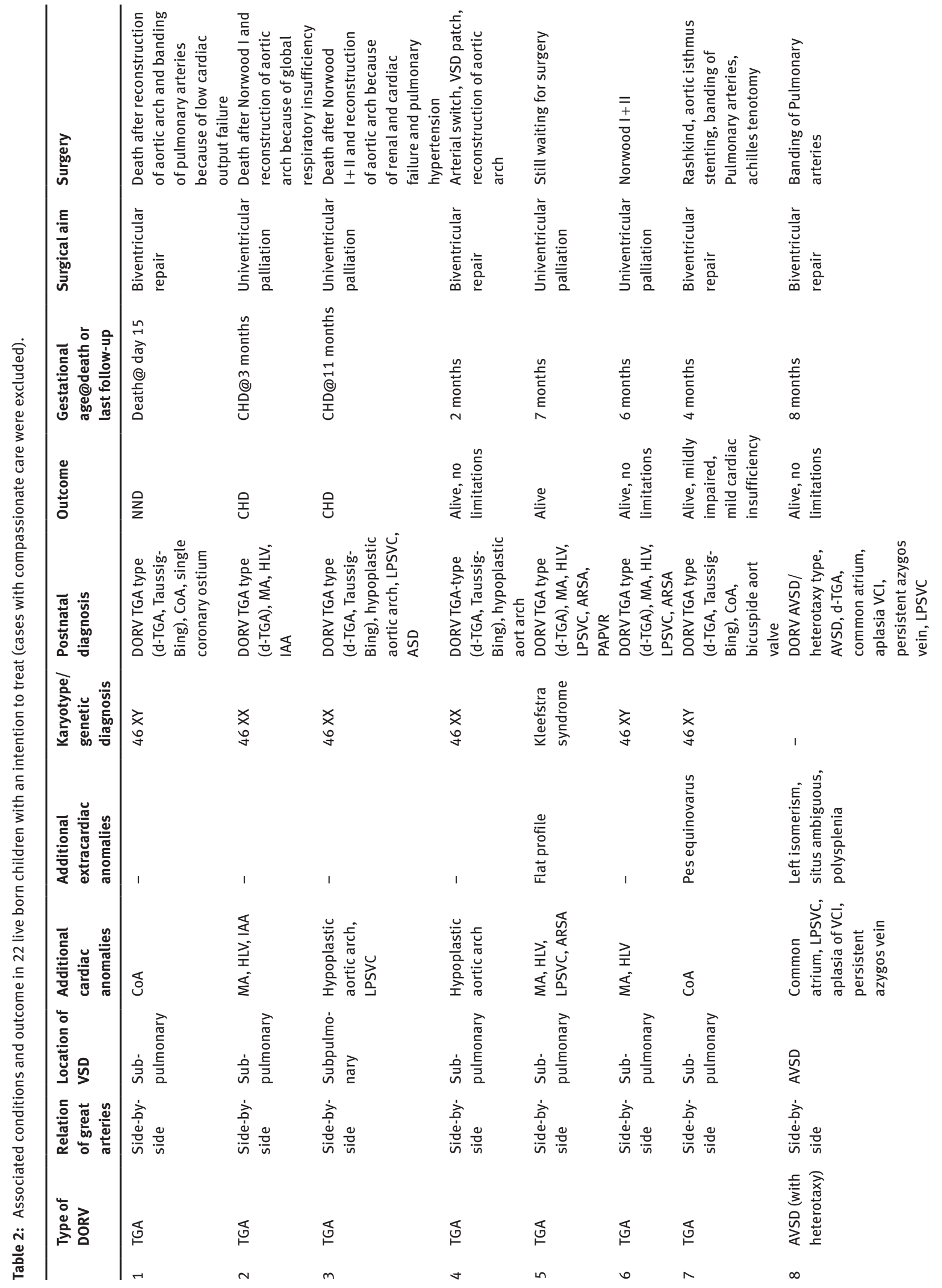




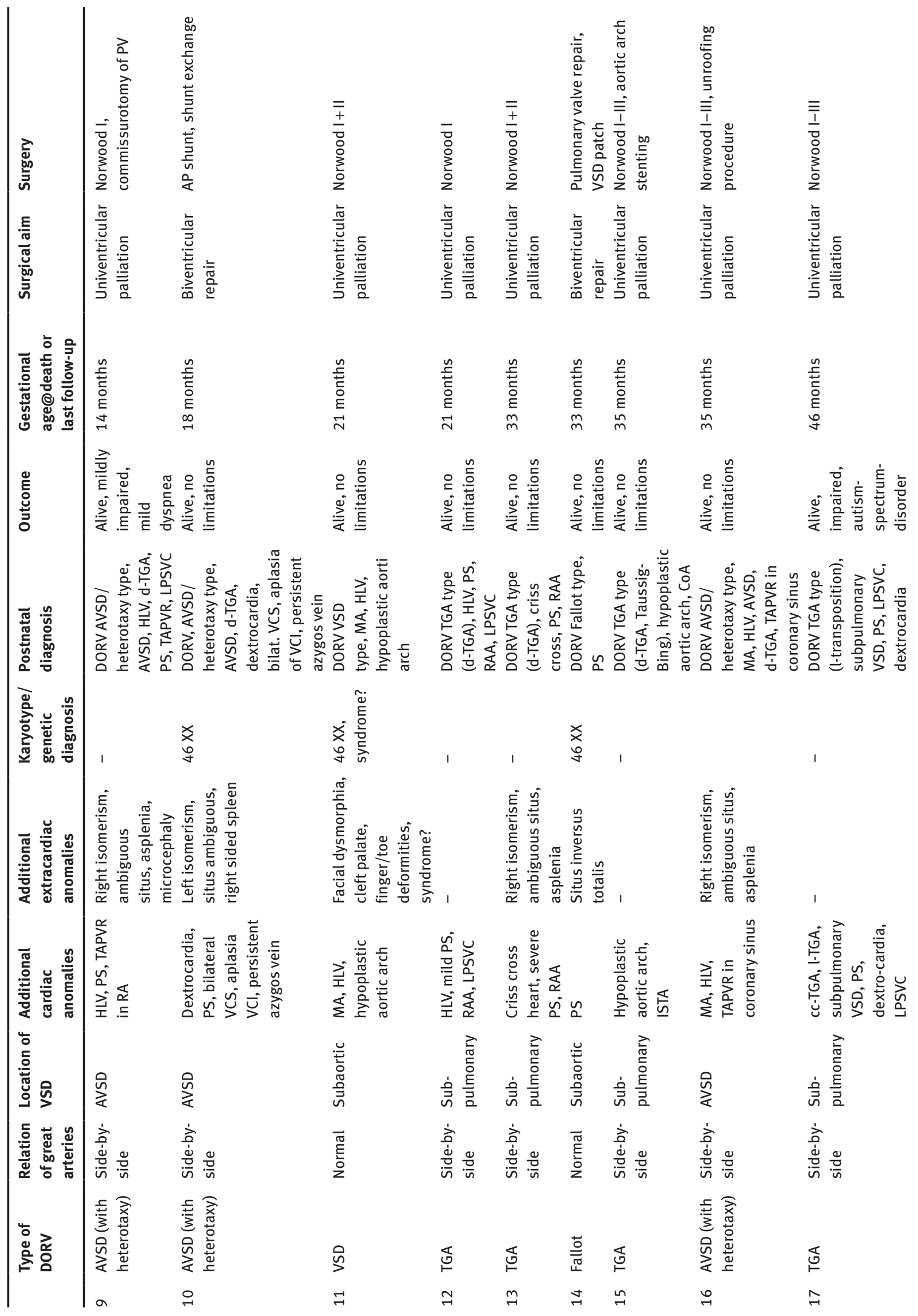




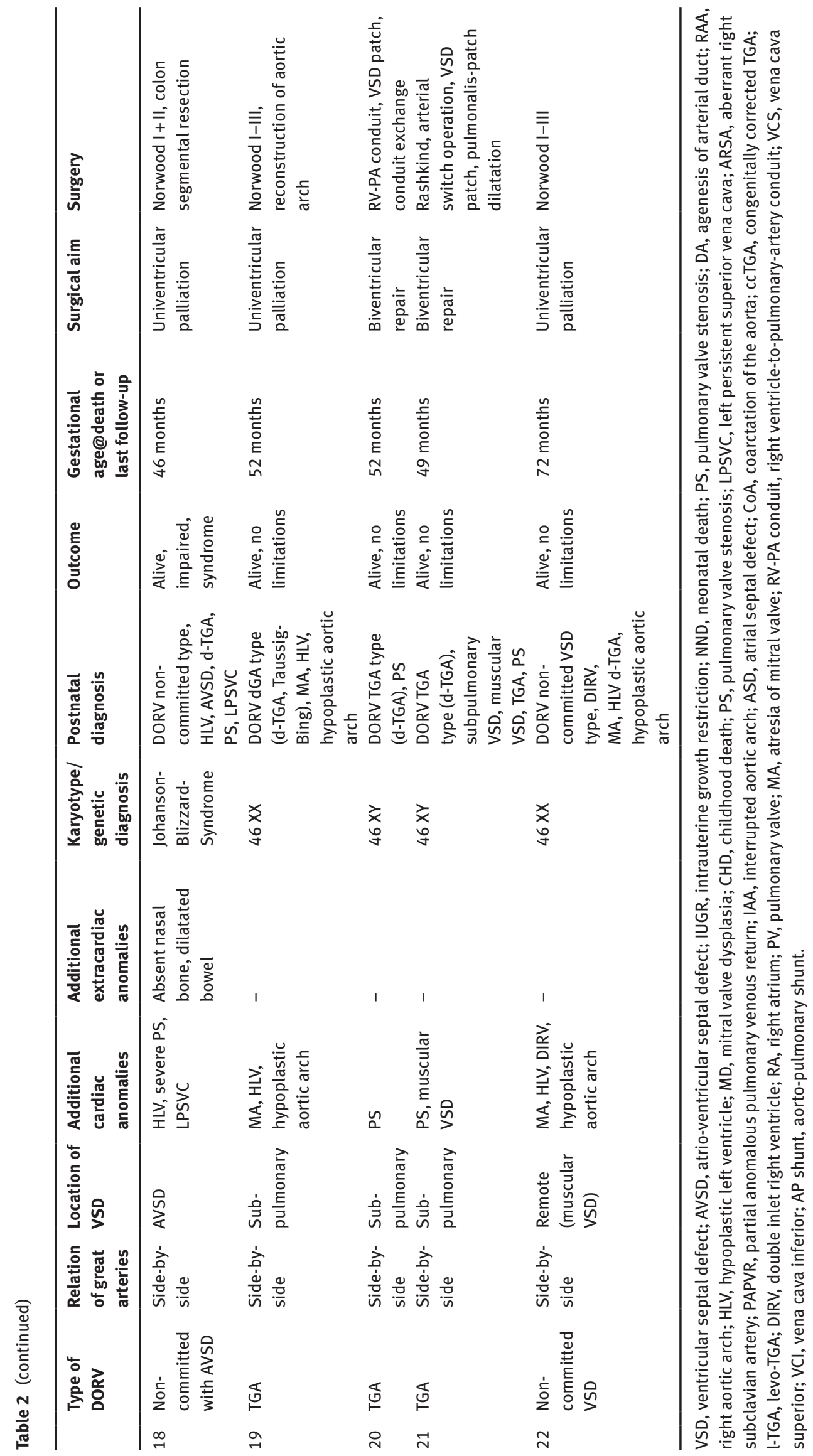




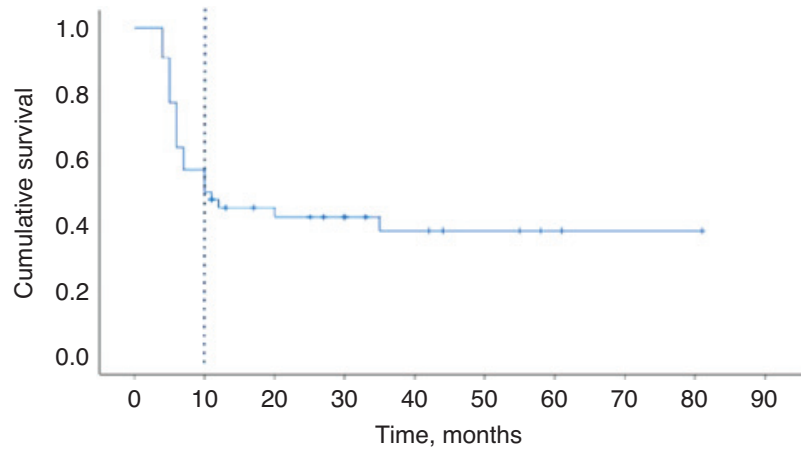

Figure 2: Kaplan-Meier curve for observed survival of 46 cases with prenatally diagnosed double outlet right ventricle.

The dotted line represents term.

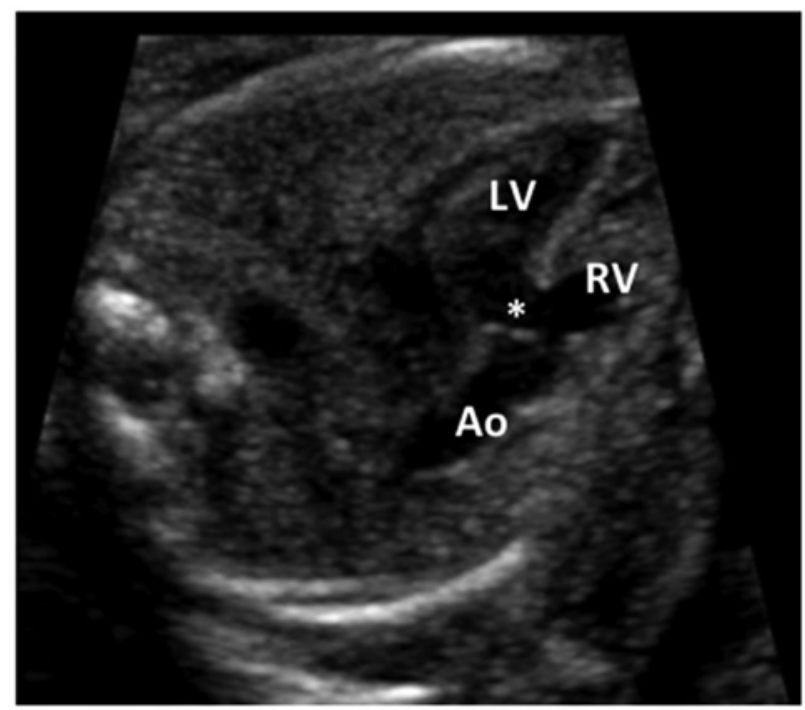

Figure 3: DORV, VSD type, with subaortic VSD and overriding aorta. $\mathrm{LV}$, left ventricle; RV, right ventricle; Ao, overriding aorta; * ${ }^{\text {subaortic }}$ VSD.

\section{Accuracy of prenatal ultrasound}

To determine the accuracy of prenatal ultrasound, we only included the 27 liveborns with available postnatal confirmation of diagnosis. A correct prenatal diagnosis of DORV was made in $96.3 \%$ (all but one) of the cases. One case of prenatal diagnosis of TGA-type DORV was postnatally classified as d-TGA with subpulmonary VSD.

In another case with Kleefstra syndrome and DORV with MA, HLV, d-TGA and subpulmonary VSD, additional partial anomalous pulmonary venous connection was missed at the prenatal ultrasound. Thus, the prenatal diagnosis was correct in $92.7 \%$ (all but two) of the cases, if the spatial relationship of the great arteries, position of the VSD and all additional cardiac anomalies are also taken into account.

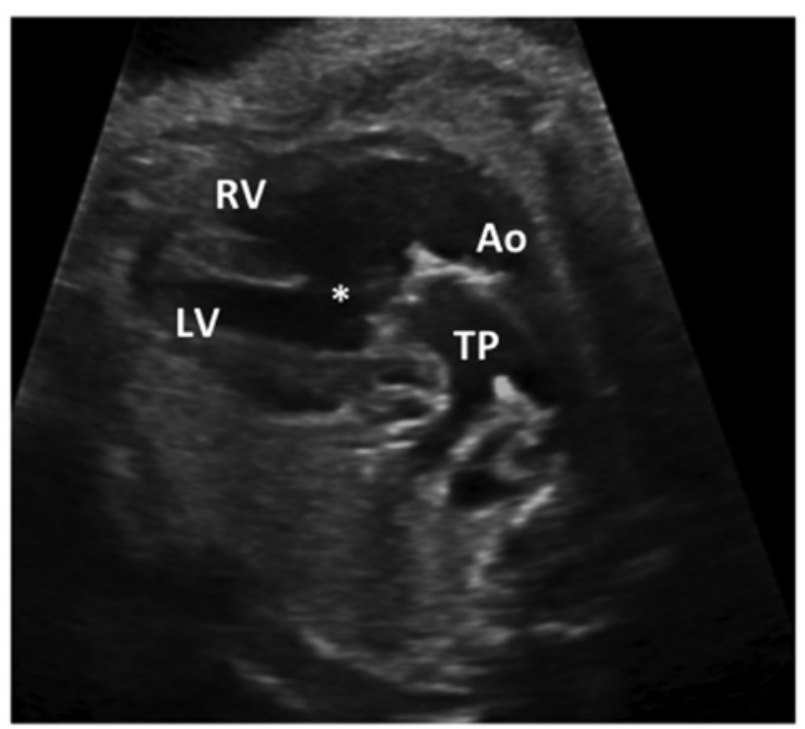

Figure 4: DORV, TGA type, with subpulmonary VSD and overriding pulmonary trunk.

LV, left ventricle; RV, right ventricle; TP, overriding pulmonary trunk; Ao, aorta; *subpulmonary VSD.

Among the 26 liveborns with a postnatally confirmed diagnosis of DORV, there were the following types of DORV: one (3.8\%) VSD type, two (7.7\%) Fallot type, 17 (65.4\%) TGA type, two (7.7\%) non-committed VSD and four (15.4\%) AVSD type with heterotaxy (Figures 3 and 4).

\section{Discussion}

DORV occurs with a prevalence of $0.46-8.8 \%$ in prenatal series and decreases to $0.003-0.02 \%$ in live-born infants $[2,9,11,22-25]$. However, only a few series of prenatally diagnosed DORV with small cohorts and only short postnatal follow-up periods have been published so far [2, 4, 9, $12,19]$. In addition, the number of survivors in these series was extremely low, due to the high rate of TOP, spontaneous intrauterine loss and considerable postsurgical mortality. Therefore, most information concerning the outcome of newborns with DORV is available from postnatal series. Our current study is one of the largest cohorts of prenatally diagnosed DORV with a larger number of survivors and therefore might improve the prenatal counseling of affected couples.

DORV was reported to be associated with additional cardiac, extracardiac and chromosomal anomalies in $90 \%, 38-58 \%$ and $25 \%$, respectively $[2,7,9,10,13,14,19$, 22, 26-28]. Similarly, we also found a high prevalence of severe additional anomalies. All our fetuses (100\%) had 
additional cardiac anomalies, including severe RVOTO and LVOTO, HLV, partial or total anomalous pulmonary venous connection or coronary anomalies. Right and left outflow tract obstructions and HLV occurred with an incidence of $46 \%, 24 \%$ and $28 \%$, respectively, which is in accordance with the reported incidence $[2,4,9,19]$. Major extracardiac anomalies occurred in $66 \%$ of our fetuses and chromosomal anomalies or genetic syndromes in $28 \%$ of our fetuses. In another $11 \%$ of fetuses, we highly suspected a chromosomal aberration or genetic syndrome, although molecular diagnosis was inconclusive. Abnormalities of laterality occurred in $22 \%$, including two cases with a lethal combination of left isomerism with AVSD and complete heart block. Previous series also reported the abnormalities of laterality in $25-35 \%$ of DORV $[9,11,19]$. In conclusion, a fetal diagnosis of DORV should trigger an extensive search for additional anomalies.

The rate of TOP in previously published cohorts of DORV varies considerably between $15.8 \%$ and $43.3 \%$ [2, 9, $16,19]$. This might be due to the fact that in some studies the diagnosis was made later in pregnancy and beyond the time period in which TOP would have been a legal option. In our cohort, the rate of TOP was 37\%. Additionally, $9 \%$ of parents in our cohort chose compassionate postnatal care. Only half of all pregnancies with a prenatal diagnosis of DORV were continued with the intention of postnatal surgical treatment.

The importance of an accurate prenatal diagnosis in counseling parents with regard to prognosis and to predict the type of postnatal surgical approach is obvious. Our study confirms that DORV can be diagnosed prenatally with a high degree of accuracy. The correct diagnosis of DORV was made in $96 \%$ of the cases. Diagnosis was usually not made prior to 21 weeks of gestation, presumably due to the predominantly normal looking four-chamber view that conceals this defect from basic cardiac screening. As basic cardiac screening in Germany does not include the assessment of left and right long axis views, the detection rate of cardiac anomalies with a normal looking four-chamber view is low. If the relationship of the great arteries, the position of VSD and all cardiac anomalies were taken into account, an accurate diagnosis was achieved in $92 \%$ of the cases. This is in accordance with previously published studies with an overall accuracy between 59 and $88 \%$ [2, $9,11,12,19]$. Despite some inaccuracy in the exact diagnosis of all anatomic details of DORV, accurate counseling regarding the type of expected surgical approach and postoperative uni- or biventricular endpoint was possible in all cases. All children predicted prenatally to need a single ventricle palliation, and all children predicted to achieve biventricular repair, ultimately received the predicted type of surgery. Except for Gelehrter et al., who achieved a correct prediction of surgical approach in $91 \%$ of cases, and Zidere et al., who achieved a correct prediction in all of their six cases, none of the previously published studies correlated the diagnostic accuracy with the prediction of postnatal surgery [11, 29].

Previously published data described a varying but overall poor prognosis of fetuses with DORV, with overall survival rates of only $21 \%-43 \%[4,9,12,19]$. Even after the exclusion of terminated pregnancies, the intentionto-treat survival rates did not exceed $50 \%$ in most cohorts and post-surgical mortality was high with up to $75 \%$ [2, $9,15,30]$. Two other studies described significantly better intention-to-treat survival rates of $63 \%$ and $69 \%$ with lower post-surgical mortality rates of $31 \%[4,19]$. Our study confirms a better prognosis with an intention-totreat survival rate of $86 \%$ and a much lower post-surgical mortality rate of $14 \%$ in our cohort. The discrepancy of survival rates may be explained by the small cohorts in all previously published studies with no more than 13 children who underwent surgery, but keeping in mind that outcome in our study was assessed at different stages of surgical repair or palliation. Not all included children had completed surgical repair or last step of palliation and postoperative follow-up periods differed significantly in our children. The majority of our survivors $(62 \%)$ ended up having univentricular palliation, only 38\% achieved biventricular repair. This is in accordance with previous reports in which up to $75 \%$ of survivors received univentricular palliation $[2,4,9,19]$.

Our study also confirms a better postsurgical health status. A total of $77.8 \%$ of our survivors were healthy without impairment after cardiac surgery at last followup. Among the four impaired children, one child was impaired because of non-cardiac problems due to a concomitant syndromal anomaly. Another child developed autism-spectrum disorder and the remaining two children were impaired only by mild dyspnea and mild cardiac insufficiency, respectively. The only previously published study that assessed the postsurgical health status reported $67 \%$ healthy survivors [4].

The strength of our single center study is that so far this is the largest published cohort of prenatally diagnosed DORV with 45 prenatally diagnosed cases, 26 cases with a postnatally confirmed diagnosis, 21 children who underwent surgery with known outcome and 18 survivors after surgery at last follow-up.

Our study also has a number of limitations. Despite our relatively large cohort, the number of newborns who underwent surgery is still small due to the rarity of the cardiac defect itself and the high percentage of TOP and 
cases in which parents declined surgical intervention postnatally. The small size of our cohort did not allow for a detailed subgroup analysis and for identifying any predictors of outcome. In addition, not all children have yet completed their ultimate surgical repair or palliation and the outcome was assessed at different time points of surgical repair or palliation, and a long-term follow-up is lacking. A further limitation is its retrospective design, which limits the assessment of detailed spatial relationship of some cardiac strutures.

In conclusion, the overall outcome strongly depends on the presence and severity of additional anomalies. In fetuses without lethal associated anomalies, the prognosis seems to be better than that previously reported with a survival rate of $>80 \%$ and a good chance of healthy shortterm survival without significant impairment, although $60 \%$ undergo univentricular palliation. In addition, postnatal type of surgical repair (uni- or biventricular) can be predicted with high accuracy on a prenatal ultrasound.

Author contributions: All the authors have accepted responsibility for the entire content of this submitted manuscript and approved submission.

Research funding: None declared.

Employment or leadership: None declared.

Honorarium: None declared.

Competing interests: The funding organization(s) played no role in the study design; in the collection, analysis, and interpretation of data; in the writing of the report; or in the decision to submit the report for publication.

\section{References}

1. Hagler DJ, Ritter DG, Puga FJ. Double-outlet right ventricle, $4^{\text {th }}$ ed. In: Adams FH, Emmanouilides GC, Riemenschneider TA, editors. Moss' heart disease in children and adolescents. Baltimore: Lippincott Williams \& Wilkins; 1989. p. 19.

2. Gedikbasi A, Oztarhan K, Gul A, Sargin A, Ceylan Y. Diagnosis and prognosis in double-outlet right ventricle. Am J Perinatol 2008;25:427-34.

3. Witham AC. Double outlet right ventricle: a partial transposition complex. Am Heart J 1957;53:928.

4. Hartge DR, Niemeyer L, Axt-Fliedner R, Krapp M, Gembruch U, Germer U, et al. Prenatal detection and postnatal management of double outlet right ventricle (DORV) in 21 singleton pregnancies. J Matern Fetal Neonatal Med 2012;25:58-63.

5. Lev M, Bharati S, Meng L, Liberthson RR, Paul MH, Idriss F. A concept of double outlet right ventricle. J Thorac Cardiovasc Surg 1972;64:2712.

6. Anderson R, Wilkinson JL, Arnold R, Becker AE, Lubkiewicz K. Morphogenesis of bulboventricular malformations. II. Observations on malformed hearts. Br Heart J 1974;36:948.
7. Walters HL III, Mavroudis C, Tchervenkov Cl, Jacobs JP, LacourGayet F, Jacobs ML. Congenital Heart Surgery Nomenclature and Database Project: double outlet right ventricle. Ann Thorac Surg 2000;69:249-63.

8. Mahle WT, Martinez R, Silverman N, Cohen MS, Anderson RH. Anatomy, echocardiography, and surgical approach to double outlet right ventricle. Cardiol Young 2008;18:39-51.

9. Kim N, Friedberg MK, Silverman NH. Diagnosis and prognosis of fetuses with double outlet right ventricle. Prenat Diagn 2006;26:740-5.

10. Lacour-Gayet F. Intracardiac repair of double outlet right ventricle. Semin Thorac Cardiovasc Surg Pediatr Card Surg Annu 2008;1:39-43.

11. Gelehrter S, Owens ST, Russell MW, van der Velde ME, GomezFifer C. Accuracy of the fetal echocardiogram in double-outlet right ventricle. Congenit Heart Dis 2007;2:32-7.

12. Sivanandam S, Glickstein JS, Pritz BF, Allan LD, Altmann K, Solowiejczyk DE, et al. Prenatal diagnosis of conotruncal malformations: diagnostic accuracy, outcome, chromosomal abnormalities and extracardiac anomalies. Am J Perinatol 2006;23:241-6.

13. Chaoui R, Korner, H, Dietel C, Tennstedt C. Spectrum of congenital heart defects and extracardiac malformations associated with chromosomal abnormalities: results of a seven year necropsy study. Heart 1999;82:34.

14. Franklin RC, Anderson R, Daniels O, Elliot MJ, Gewillig MH, Ghisla R, et al. Report of the Coding Committee of the Association for European Paediatric Cardiology. Cardiol Young 2002;12:611-8.

15. Allan LD, Sharland GK, Milburn A, Lockhart SM, Groves AM, Anderson $\mathrm{RH}$, et al. Prospective diagnosis of 1,006 consecutive cases of congenital heart disease in the fetus. J Am Coll Cardiol 1994;23:1452-8.

16. Brick DH, Allan LD. Outcome of prenatally diagnosed congenital heart disease: an update. Pediatr Cardiol 2002;23:449-53.

17. Paladini D, Rustico M, Todros T, Palmeri S, Gaglioti P, Bennetoni $A$, et al. Conotruncal anomalies in prenatal life. Ultrasound Obstet Gynecol 1996;8:241-6.

18. Fesslova V, Nava S, Villa L. Evolution and long term outcome in cases with fetal diagnosis of congenital heart disease: Italian multicentre study. Fetal Cardiology Study Group of the Italian Society of Pediatric Cardiology. Heart 1999;82:594-9.

19. Smith RS, Comstock CH, Kirk JS, Lee W, Riggs T, Weinhouse E. Double-outlet right ventricle: an antenatal diagnostic dilemma. Ultrasound Obstet Gynecol 1999;14:315-9.

20. Yagel S, Cohen SM, Achiron R. Examination of the fetal heart by five short-axis views: a proposed screening method for comprehensive cardiac evaluation. Ultrasound Obstet Gynecol 2001;17:367-9.

21. Carvalho JS, Ho SY, Shinebourne EA. Sequential segmental analysis in complex fetal cardiac abnormalities: a logical approach to diagnosis. Ultrasound Obstet Gynecol 2005;26:105-11.

22. Wu M-H, Chen H-C, Lu C-W, Wang J-K, Huang S-C, Huang S-K. Prevalence of congenital heart disease at live birth in Taiwan. J Pediatr 2010;156:782-5.

23. Wang NK, Shen CT, Lin MS. Results of echocardiographic screening in 10000 newborns. Acta Paediatr Taiwan 2007;48:7-9.

24. Hoffman JIE, Kaplan S. The incidence of congenital heart disease. J Am Coll Cardiol 2002;39:1890-900. 
25. Friedberg MK, Silverman NH. Changing indications for fetal echocardiography in a University Center population. Prenat diagn 2004;24:781-6.

26. Khositseth A, Tocharoentanaphol C, Khowsathit P, Ruangdaraganon N. Chromosome 22q11 deletions in patients with conotruncal heart defects. Pediatr Cardiol 2005; 26:570-3.

27. Pitkänen OM, Hornberger LK, Miner SES, Mondal T, Smallhorn JF, Jaeggi E, et al. Borderline left ventricles in prenatally diagnosed atrioventricular septal defect or double outlet right ventricle: echocardiographic predictors of biventricular repair. Am Heart J 2006;152:163.e1-7.
28. Axt-Fliedner R, Hartge D, Krapp M, Berg C, Geipel A, Koester $S$, et al. Course and outcome of fetuses suspected of having coarctation of the aorta during gestation. Ultraschall Med 2009;30:269-76.

29. Zidere V, Pushparajah K, Allan LD, Simpson JM. Three-dimensional fetal echocardiography for prediction of postnatal surgical approach in double outlet right ventricle: a pilot study. Ultrasound Obstet Gynecol 2013;23:421-5.

30. Tometzki AJ, Suda K, Kohl T, Kovalchin JP, Silverman NH. Accuracy of prenatal echocardiographic diagnosis and prognosis of fetuses with conotruncal anomalies. J Am Coll Cardiol 1999;33:1696-701. 